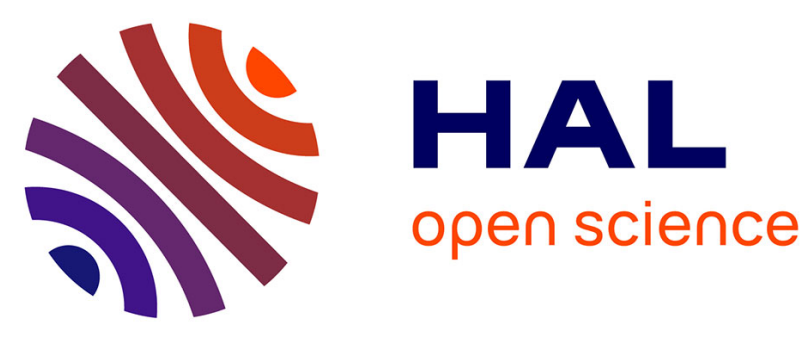

\title{
Microcavity-quality-factor enhancement using nonlinear effects close to the bistability threshold and coherent population oscillations
}

\author{
Yannick Dumeige, Alejandro M. Yacomotti, Patricio Grinberg, Kamel \\ Bencheikh, Elodie Le Cren, Juan Ariel Levenson
}

\section{To cite this version:}

Yannick Dumeige, Alejandro M. Yacomotti, Patricio Grinberg, Kamel Bencheikh, Elodie Le Cren, et al.. Microcavity-quality-factor enhancement using nonlinear effects close to the bistability threshold and coherent population oscillations. Physical Review A: Atomic, molecular, and optical physics [1990-2015], 2012, 85 (6), pp.063824. 10.1103/PhysRevA.85.063824 . hal-00840654

\section{HAL Id: hal-00840654 https://hal.science/hal-00840654}

Submitted on 7 Oct 2013

HAL is a multi-disciplinary open access archive for the deposit and dissemination of scientific research documents, whether they are published or not. The documents may come from teaching and research institutions in France or abroad, or from public or private research centers.
L'archive ouverte pluridisciplinaire HAL, est destinée au dépôt et à la diffusion de documents scientifiques de niveau recherche, publiés ou non, émanant des établissements d'enseignement et de recherche français ou étrangers, des laboratoires publics ou privés. 


\title{
Microcavity quality factor enhancement using nonlinear effects close to the bistability threshold and coherent population oscillations
}

\author{
Y. Dumeige, ${ }^{1,2, *}$ A. M. Yacomotti, ${ }^{3}$ P. Grinberg, ${ }^{3}$ K. Bencheikh, ${ }^{3}$ E. Le Cren, ${ }^{1,2}$ and J. A. Levenson ${ }^{3}$ \\ ${ }^{1}$ UEB, Université Européenne de Bretagne, Université de Rennes I, France \\ ${ }^{2}$ CNRS, UMR 6082 FOTON, Enssat, 6 rue de Kerampont, BP 80518, 22305 Lannion cedex, France \\ ${ }^{3}$ Laboratoire de Photonique et de Nanostructures CNRS-UPR020, Route de Nozay, F-91460 Marcoussis, France
}

(Dated: February 13, 2013)

\begin{abstract}
We analytically show that inserting a driven two level system inside a microcavity can improve its optical properties. In this approach, the strong dispersion induced by a pump via population oscillations increases the cavity lifetime experienced by a slightly detuned probe. We further predict that if the cavity is pumped through a resonant channel, optical absorptive or dispersive bistability can be combined with the population oscillation induced steep material dispersion to obtain a strong quality factor enhancement. Moreover differential amplification coming from the nonlinear feature of the pump transfer function can be used to drastically increase the probe transmission beyond intrinsic characteristics of the resonator. The Q-factor enhancement and the differential amplification can be advantageously combined with a frequency pulling effect to stabilize or readjust the microcavity resonance frequency.
\end{abstract}

PACS numbers: 42.55.Sa, 42.65.Pc, 42.50.Gy

Keywords: Cavity lifetime, Bistability, Population Oscillations, Microcavity

\section{INTRODUCTION}

Microcavities with high quality (Q) factors are of great interest for integration of various optical functions and fundamental studies in optical physics [1-3]. The narrow resonance and the long photon lifetime of such devices can be used in optical and microwave filtering applications $[4,5]$ or for optical buffer miniaturization [6]. Moreover devices with adjustable Q-factors from low to high values can be exploited to break fundamental limits in pulse storing systems [7-9].

Intrinsic limits for the Q-factor in a microcavity are given either by its radiative losses due to imperfect light confinement or by the residual absorption of the constituting material. Therefore, the manufacturing of high-Q resonators requires high purity materials [10], complex technological processes to reduce fabrication imperfections [11] and careful design to avoid radiative losses $[12,13]$. It is possible to compensate for optical losses by using a gain material within the microcavity [14-16]. Another way to increase the Q-factor consists in inserting a highly dispersive material inside the microcavity [17]. In this case, the photon lifetime is increased by a factor proportional to the group index of the dispersive material $[18,19]$. This technique has already been proposed and experimentally demonstrated in atomic systems embedded in macroscopic ring cavities using coherent effects such as coherent population trapping or electromagnetically induced transparency (EIT) [18-23]. In those configurations, a powerful pump beam induces a steep dispersion centered at the signal frequency, tuned to the cavity resonance. In this context, it has also been

* yannick.dumeige@univ-rennes1.fr shown that a nonlinear susceptibility at the probe frequency can enhance the Q-factor of the resonator [24]. The Q-factor of a solid state whispering gallery mode microresonator has been recently greatly improved using the dispersion induced by optomechanical effects [25]. From another point of view, a strong dispersion can also been obtained in solid state configurations via coherent population oscillations (CPO) with any two level systems (TLS) including ion doped crystals or semiconductor materials under suitable pumping [26-29]. Very few papers have reported on the coupling of material dispersion or coherent effects and microcavity resonances to artificially enhance the group delay achievable in semiconductor heterostructures [30-34] or to control the Q-factor [35]. Note that CPO effect occurring inside a semiconductor laser cavity have been recently analyzed [36].

In this paper we discuss the coupling of an active (semiconductor or atomic) medium to an optical cavity (or microcavity) where both the pump and the probe beams are resonant. We will show that the nonlinear response of the pump induces an additional dispersion which can increase the Q-factor of the cavity at the probe frequency. Moreover, under particular conditions, the pump can also induce a strong differential gain [37] on the probe, thus improving the cavity transmission. This paper is organized as follows. In section II we use a simple approach to explain the Q-factor enhancement by CPO effect considering that only the probe beam is solely resonantly coupled to the cavity. The model based on the coupled mode theory (CMT) which describes the nonlinear cavity is worked out in detail at section III. With this approach, the nonlinear response of the cavity regarding the pump beam can be taken into account. Section IV is devoted to analytic calculations enabling us to identify the physical mechanisms leading to Q-factor enhancement in a nonlinear microcavity. Finally, at section $\mathrm{V}$ we show some 
numerical results illustrating the cavity linewidth narrowing, the frequency pulling of the resonance frequency and the role played by population oscillations and optical bistability in these effects.

\section{BASIC APPROACH OF CPO IN A MICROCAVITY}

Figure 1 is a schematic of a microcavity containing a medium where population oscillations can occur. This medium can be ion doped crystals [26, 28], or semiconductor heterostructures [29] for example. The following analysis based on CMT [38] is well adapted to several configurations including whispering gallery mode resonators or photonic crystal microcavities [39]. The ab-

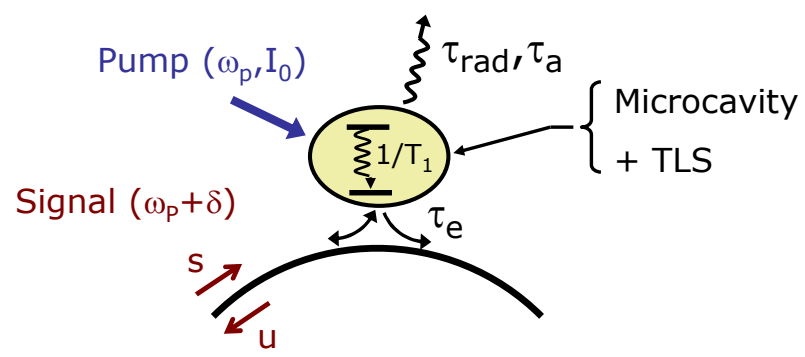

FIG. 1. (Color online) Microcavity containing a TLS. The pump (frequency $\omega_{P}$ ) is non-resonantly coupled to the cavity. $s$ (frequency $\omega_{s}=\omega_{P}+\delta$ ) is the input probe field. The cavity mode is coupled to the reflected field $u$ via evanescent waves with a characteristic lifetime $\tau_{e}$.

sorption coefficient of the medium $\alpha$ can be saturated using a strong pump field (characterized by an angular frequency $\omega_{P}$ and a normalized intensity $I_{0}=I / I_{\text {sat }}$ where $I$ and $I_{\text {sat }}$ are the pump and saturation intensities). The probe field $s$ with the angular frequency $\omega_{s}=\omega_{P}+\delta$, is coupled into the cavity with a characteristic time $\tau_{e}$. For simplicity we consider that the probe beam is at cavity resonance: $\omega_{0}=\omega_{s}$. In this first approach only the signal is resonantly coupled to the cavity. The pump is coupled using a non-resonant channel such as single pass vertical pumping in a photonic crystal cavity or whispering gallery mode microcavity for example. The cavity mode is coupled back into the access line via two channels (see Fig. 1), and we only consider the reflected field $u$. The overall photon lifetime in the cavity is given by

$$
\frac{1}{\tau}=\frac{2}{\tau_{e}}+\frac{1}{\tau_{\text {rad }}}+\frac{1}{\tau_{a}},
$$

where $\tau_{\text {rad }}$ is the cavity lifetime limited by the radiation losses. $\tau_{a}$ is related to the absorption coefficient $\alpha$ by:

$$
\frac{1}{\tau_{a}}=\frac{c \alpha}{2 n_{0}}
$$

with $n_{0}$ the effective refractive index of the material constituting the cavity. For a pump/probe detuning $\delta<1 / T_{1}$, where $T_{1}$ is the population lifetime, the population oscillation effect generates a strong dispersion on the probe beam characterized by a group index $n_{g}$ calculated from a density matrix approach (at resonance $\delta=0$ ) assuming a short dipole moment dephasing time $[26,40]$

$$
n_{g}=\frac{\alpha_{0} c T_{1}}{2} \frac{I_{0}}{\left(1+I_{0}\right)^{3}}
$$

and a saturation of the absorption leading to

$$
\alpha=\frac{\alpha_{0}}{\left(1+I_{0}\right)^{2}}
$$

where $\alpha_{0}$ is the unsaturated absorption. If $n_{g} \gg n_{0}$, the quality factor of the cavity experienced by the probe beam is thus given by $[17,18,41,42]$ :

$$
Q=\frac{n_{g}}{n_{0}} \frac{\omega_{0} \tau}{2}
$$

which can also be written using Eqs. (1-5)

$$
Q=\frac{\omega_{0} T_{1} I_{0}}{2\left(1+I_{0}\right)\left[1+\tau_{a 0}\left(\frac{2}{\tau_{e}}+\frac{1}{\tau_{\text {rad }}}\right)\left(1+I_{0}\right)^{2}\right]},
$$

where

$$
\frac{1}{\tau_{a 0}}=\frac{c \alpha_{0}}{2 n_{0}}
$$

We now consider two different configurations:

- The strong unsaturated absorption: $\tau_{e}, \tau_{\text {rad }} \gg \tau_{a 0}$. In this case the overall Q-factor can be approximated by

$$
Q=\frac{\omega_{0} T_{1} I_{0}}{2\left(1+I_{0}\right)} .
$$

This expression shows that the new lifetime of the cavity is $T_{1} I_{0} /\left(1+I_{0}\right)$. Choosing $T_{1} \gg \tau_{\text {rad }}$, the population oscillation greatly increases the cavity lifetime which maximal value is $\tau_{\text {rad }}$ when all the other optical sources are negligible. The only drawback of this configuration is that the value of the reflected power given by

$$
\left|\frac{u}{s}\right|^{2}=\left(\frac{2 \tau_{a 0}}{\tau_{e}}\right)^{2}\left(1+I_{0}\right)^{4}
$$

can be very weak for $\tau_{a 0} \ll \tau_{e}$.

- The weak unsaturated absorption: $\tau_{e}, \tau_{\text {rad }} \ll \tau_{a 0}$. The Q-factor can now be written as

$$
Q=\frac{T_{1}}{\tau_{a 0}} \frac{I_{0}}{\left(1+I_{0}\right)^{3}} Q_{0},
$$

where the Q-factor of the cold cavity $Q_{0}=\omega_{0} \tau_{0} / 2$ is deduced from the photon lifetime of the cold cavity

$$
\frac{1}{\tau_{0}}=\frac{2}{\tau_{e}}+\frac{1}{\tau_{\text {rad }}}+\frac{1}{\tau_{a 0}} .
$$


Considering the hypothesis, we get

$$
\frac{1}{\tau_{0}} \approx \frac{2}{\tau_{e}}+\frac{1}{\tau_{\text {rad }}}
$$

The main limitation of this approach is that the Q-factor is proportional to $T_{1} / \tau_{a 0}$ which is limited since we have assumed a high value of $\tau_{a 0}$. This leads to a moderate value of $\mathrm{CPO}$ enhanced $\mathrm{Q}$-factor.

As a partial conclusion, we point out that the cavity photon lifetime of a microcavity containing a TLS where CPO occurs can be strongly increased. The cavity lifetime is then basically limited by the population lifetime $T_{1}$. However, unlike other coherent effect based schemes where absorption is completely canceled, in the CPO approach, the residual unsaturated absorption limits the reflected power from the cavity. We will show in next sections that these issues can be circumvented taking advantage of the nonlinear properties of the cavity.

\section{MODEL FOR POPULATION OSCILLATION IN A NONLINEAR MICROCAVITY}

In this section we study the case where both the probe and the pump beams are resonant with the cavity mode. It has already been shown that the CPO effects can be explained in the framework of a saturable absorber system [43-47]. Since the CMT is well adapted to the modeling of nonlinear single mode microcavities [48-50], we apply this approach to our system composed of an absorbing semiconductor material playing the role of the TLS [51]. The mode amplitude in the cavity $a(t)$ is described by a

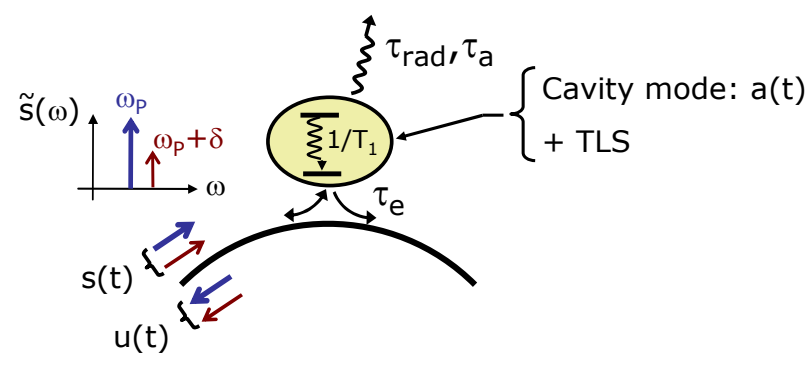

FIG. 2. (Color online) Nonlinear microcavity and its access line. $s(t)$ is the input field consisting of a pump and a signal which carrier frequencies are respectively $\omega_{P}$ and $\omega_{s}=\omega_{P}+$ $\delta(\tilde{s}(\omega)$ represents the input signal spectrum). The cavity mode $a(t)$ is coupled to $s(t)$ and to the reflected field $u(t)$ via evanescent waves with a characteristic lifetime $\tau_{e}$.

simple harmonic oscillator model [38]

$$
\frac{d a}{d t}=\left[j\left(\omega_{0}+\Delta \omega\right)-\frac{1}{\tau}\right] a(t)+\sqrt{\frac{2}{\tau_{e}}} s(t)
$$

where $s(t)$ is the input field. Note that $|a(t)|^{2}$ is the energy stored inside the cavity and the power of the input signal is given by $|s(t)|^{2}$. We assume that the absorption linearly depends on the carrier density $N$

$$
\alpha=\alpha_{0} \cdot\left(1-\frac{N}{N_{t}}\right)
$$

where $N_{t}$ is the carrier density at transparency. The index variations due to the photocreated carriers induce a resonance frequency shift $\Delta \omega$ of the cavity. The absorption and the index variations are related through the Henry's linewidth enhancement factor $\alpha_{H}$ [52]. With these definitions, $\omega_{0}$ is the resonance frequency of the cavity at transparency $\left(N=N_{t}\right)$. The frequency shift due to carrier density variations is $[53-56]$

$$
\Delta \omega=\frac{\alpha_{H}}{\tau_{a 0}} \cdot\left(\frac{N}{N_{t}}-1\right) .
$$

The output field $u(t)$ is obtained from the cavity mode amplitude expression

$$
u(t)=\sqrt{\frac{2}{\tau_{e}}} a(t)
$$

Considering the time domain slowly varying envelope approximation, the definition of the pump angular frequency $\omega_{P}$ and the expression of the field amplitudes

$$
\begin{aligned}
a(t) & =A(t) e^{j \omega_{P} t} \\
s(t) & =S(t) e^{j \omega_{P} t} \\
u(t) & =U(t) e^{j \omega_{P} t}
\end{aligned}
$$

Eq. (13) can be recast as

$$
\begin{aligned}
\frac{d A}{d t}= & {\left[j \Delta-\frac{1}{\tau_{0}}\right.} \\
& \left.+\frac{N}{N_{t} \tau_{a 0}}\left(1+j \alpha_{H}\right)\right] A(t)+\sqrt{\frac{2}{\tau_{e}}} S(t)
\end{aligned}
$$

where $\Delta$ is the detuning between the pump frequency and the cold cavity resonance frequency

$$
\Delta=\omega_{0}-\frac{\alpha_{H}}{\tau_{a 0}}-\omega_{P}
$$

The carrier density is simultaneously obtained by solving the differential equation $[57,58]$

$$
\frac{d N}{d t}=-\frac{N(t)}{T_{1}}-\frac{|A(t)|^{2}}{T_{1}\left|a_{\text {sat }}\right|^{2}}\left[N(t)-N_{t}\right]
$$

where $\left|a_{\text {sat }}\right|^{2}$ is the saturation energy and $T_{1}$ the carrier lifetime. The last equation can also be derived from the effective TLS model for semiconductor materials [51] by assuming a short polarization lifetime. This simple model could also be applied to a TLS [44], in this case the Henry's factor $\alpha_{H}$ would be replaced by the detuning between the atomic resonance frequency and the pump frequency. 


\section{ANALYTICAL RESULTS}

\section{A. First order calculations}

We now consider an input field consisting of a strong pump $s_{0}$ and a weak probe signal $s_{1}$ (with $\left|s_{1}\right| \ll\left|s_{0}\right|$ ) slightly detuned from the pump frequency

$$
S(t)=s_{0}+s_{1} e^{j \delta t} .
$$

The cavity mode and the carrier density can be expanded in a similar way

$$
\begin{aligned}
& A(t)=a_{0}+a_{1} e^{j \delta t}+a_{-1} e^{-j \delta t} \\
& U(t)=u_{0}+u_{1} e^{j \delta t}+u_{-1} e^{-j \delta t} \\
& N(t)=N_{0}+N_{1} e^{j \delta t}+N_{-1} e^{-j \delta t} .
\end{aligned}
$$

Due to the nonlinear term in Eq. (20) we have to take into account the frequency component in $-\delta$ for the cavity mode, the output field and the carrier density. By combining these expressions with Eq. (18) and identifying the zero and first order terms, we obtain the three following equalities

$$
\begin{aligned}
0= & \left(j \Delta-\frac{1}{\tau_{0}}\right) a_{0} \\
& +\frac{N_{0}\left(1+j \alpha_{H}\right)}{N_{t} \tau_{a 0}} a_{0}+\sqrt{\frac{2}{\tau_{e}}} s_{0} \\
j \delta a_{1}= & \left(j \Delta-\frac{1}{\tau_{0}}\right) a_{1} \\
& +\frac{1+j \alpha_{H}}{N_{t} \tau_{a 0}}\left(N_{0} a_{1}+N_{1} a_{0}\right)+\sqrt{\frac{2}{\tau_{e}}} s_{1} \\
-j \delta a_{-1}= & \left(j \Delta-\frac{1}{\tau_{0}}\right) a_{-1} \\
& +\frac{1+j \alpha_{H}}{N_{t} \tau_{a 0}}\left(N_{0} a_{-1}+N_{-1} a_{0}\right) .
\end{aligned}
$$

The same operation can be done using Eq. (20), we thus obtain

$$
\begin{aligned}
0= & -\frac{N_{0}}{T_{1}}-\frac{\left|a_{0}\right|^{2}}{T_{1}\left|a_{\text {sat }}\right|^{2}}\left(N_{0}-N_{t}\right) \\
j \delta N_{1}= & -\frac{N_{1}}{T_{1}}-\frac{1}{T_{1}\left|a_{\text {sat }}\right|^{2}} \\
& {\left[\left(N_{0}-N_{t}\right)\left(a_{0} a_{-1}^{*}+a_{1} a_{0}^{*}\right)+N_{1}\left|a_{0}\right|^{2}\right] } \\
-j \delta N_{-1}= & -\frac{N_{-1}}{T_{1}}-\frac{1}{T_{1}\left|a_{\text {sat }}\right|^{2}} \\
& {\left[\left(N_{0}-N_{t}\right)\left(a_{0} a_{1}^{*}+a_{-1} a_{0}^{*}\right)+N_{-1}\left|a_{0}\right|^{2}\right] . }
\end{aligned}
$$

Equation (24a) gives the relation between the normalized intracavity pump energy $x=\left|a_{0} / a_{\text {sat }}\right|^{2}$ and the static carrier density $N_{0}$,

$$
\frac{N_{0}}{N_{t}}=\frac{x}{1+x}
$$

We introduce $y=N_{0} / N_{t}$ for convenience. Combining Eq. (25) and Eq. (23a) we obtain a relation between $x$ and the pump input power $P_{\text {in }}=\left|s_{0}\right|^{2}$ :

$$
\frac{P_{i n}}{P_{0}}=\frac{x \tau_{0} \tau_{e}}{2}\left|j \Delta-\frac{1}{\tau_{0}}+\frac{x}{1+x} \cdot \frac{1+j \alpha_{H}}{\tau_{a 0}}\right|^{2},
$$

where $P_{0}=\left|a_{\text {sat }}\right|^{2} / \tau_{0}$. Basically, this relation gives the nonlinear pump transmission. Under some conditions, the equation $P_{\text {in }}=f(x)$ can have two solutions giving a bistable behavior to the cavity. Since $N(t)$ is real we have $N_{1}=N_{-1}^{*}$, thus using Eq. (23c) we find

$$
a_{-1}^{*}(\delta)=a_{0}^{*} \frac{N_{1}(\delta)}{N_{t}} H(\delta)
$$

where

$$
H(\delta)=\frac{1-j \alpha_{H}}{\eta+j(\delta+\Delta) \tau_{a 0}-y\left(1-j \alpha_{H}\right)}
$$

and $\eta=\tau_{a 0} / \tau_{0}$. Using Eq. (27), Eq. (24b) can be rewritten

$$
\frac{N_{1}}{N_{t}}=\frac{a_{1} a_{0}^{*}}{\left|a_{\text {sat }}\right|^{2}} F(\delta)
$$

where $F(\delta)$ is defined as

$$
F(\delta)=\frac{1}{1+x+j \delta T_{1}-y H(\delta)} .
$$

Substituting Eq. (29) into Eq. (23b) and using Eq. (16) we can deduce the expression of the probe amplitude reflection $r=u_{1} / s_{1}$

$$
r(\delta)=\frac{2 \tau_{a 0} / \tau_{e}}{j(\delta-\Delta) \tau_{a 0}+\eta-\left(1+j \alpha_{H}\right)[y+x F(\delta)]}
$$

\section{B. Asymptotic behavior}

In this section, we evaluate Eq. (31) at resonance $(\delta=$ $0)$ in two asymptotic cases.

\section{Purely absorptive nonlinearity $\alpha_{H}=0$}

This simple model could describe a TLS with a pump tuned to the maximum of absorption. We now assume that $\Delta=0, H(\delta)$ can thus be expressed as

$$
H(\delta)=\frac{1}{\eta-y+j \delta \tau_{a 0}} .
$$

At resonance, $\delta \rightarrow 0$ and $\left|\delta \tau_{a 0}\right| \ll \eta-y$ which leads to

$$
H(\delta)=\frac{1}{\eta-y}-\frac{j \delta \tau_{a 0}}{(\eta-y)^{2}}
$$


Substituting this last relation in Eq. (30) and making the same approximations we obtain

$$
F(\delta)=\frac{1}{(1+x)^{2}+\frac{x}{y-\eta}}+j \frac{\delta\left[\frac{T_{1}}{y-1}-\frac{\tau_{a 0} x}{(\eta-y)^{2}}\right]}{\left[(1+x)^{2}+\frac{x}{y-\eta}\right]^{2}} .
$$

With $F(\delta)=p+j \delta q$, the reflection coefficient reads

$$
r(\delta)=\frac{2 \tau_{a 0} / \tau_{e}}{\eta-y-x p+j \delta\left(\tau_{a 0}-x q\right)} .
$$

The power reflection coefficient $|r(\delta)|^{2}$ has a Lorentzian profile and the associated Q-factor can be evaluated using

$$
Q=\frac{\omega_{0}}{2}\left|\frac{\tau_{a 0}-x q}{\eta-y-x p}\right|
$$

- In the case of a long carrier lifetime: $T_{1} \gg \tau_{a 0}$, using Eq. (34) we obtain simpler expressions for $q$ and $p$ and the overall Q-factor can now be written

$$
Q=\frac{\omega_{0} T_{1}(y-\eta) x}{2(y-1)|\xi(x)|[\xi(x)+x]},
$$

where we have introduced

$$
\xi(x)=(\eta-y)(1+x)^{2}-2 x
$$

One can check that $\xi(x)+x>0$. For $\eta<9 / 8, \xi(x)$ can be null for two distinct values $x_{1}$ and $x_{2}\left(x_{1}<x_{2}\right)$ of $x$. This is the bistability condition for a nonlinear absorptive resonator [59]. At the matching values of the input pump power, the probe cavity Q-factor dramatically increases. The normalized reflected power at resonance given by

$$
|r(0)|^{2}=\left[\frac{2 \tau_{a 0}[\xi(x)+x]}{\tau_{e}(\eta-y) \xi(x)}\right]^{2},
$$

can be higher than unity since $\xi(x) \rightarrow 0$ when $x \rightarrow x_{1}$ : the signal probe is amplified. This behavior comes from a differential gain [37] experienced by the probe due to the nonlinear shape of the transmission curve of the device as illustrated at Fig. 3. One of the reasons for the Q-factor enhancement is the bistable behavior of the cavity. Indeed, in this configuration, in the bistability power zone, the pump reflectivity and phase shift have a very stiff profile. Consequently, the probe signal undergoes a strong phase shift together with a steep amplitude transmission. This leads to a strong dispersion as well as to a differential amplification. Assuming now that $\eta \approx 1$, which is possible since $1<\eta<9 / 8$, Eq. (37) can be written

$$
Q=\frac{\omega_{0} T_{1} x}{2|1-x|}
$$

This last equation explicitly shows that the Q-factor enhancement is twofold: i) as expected the photon lifetime and thus the Q-factor are proportional to $T_{1}$ and ii) for

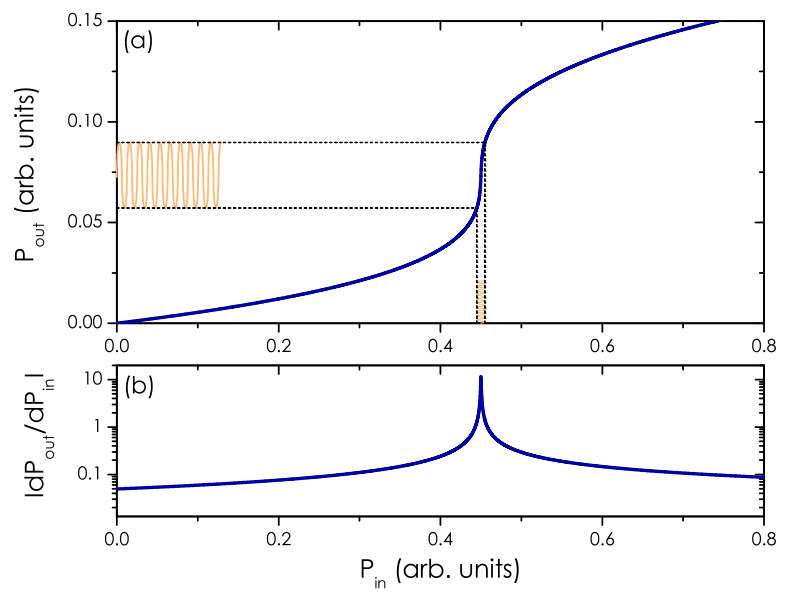

FIG. 3. (Color online) (a) Nonlinear transfer function $P_{\text {out }}=$ $f\left(P_{\text {in }}\right)$ where $P_{\text {out }}=\left|u_{0}\right|^{2}$ of a cavity just below the bistability threshold. We have also schematically sketched the transfer function of a small modulation stemming from the beating between the pump and the probe fields. (b) For input power values close to the switching power $\left(P_{i n}=0.45\right.$ in the example), the differential gain $\left|d P_{\text {out }} / d P_{\text {in }}\right|$ can be very high and can even diverge for bistable cavities.

$x \rightarrow x_{1}=1$, the Q-factor can be arbitrarily increased. Note that this analysis is valid in the small signal limit $\left(\left|a_{ \pm 1}\right| \ll\left|a_{0}\right|\right)$. In particular, for $x=1$, the pump the signal amplitude is such high that the first order approximations do not hold. The saturation of the differential amplification can be calculated by numerically solving Eqs. (18) and (20). As already discussed, the nonlinear transmission of the cavity at the pump frequency also gives a strong differential amplification which can be useful to compensate for residual losses of the cavity at the probe frequency. For $x \approx 0$ and $x \gg 1$ Eqs. (8) and (40) give the same results; the two approaches differ only when $x \approx x_{1}$ and $x \approx x_{2}$. From another point of view if $\eta \gg 1$ we can write

$$
|\xi(x)| \approx \xi(x)+x \approx \eta(1+x)^{2},
$$

noting that $y-1=-1 /(1+x)$ one can check that Eqs. (8) and (37) lead to the same expression of the Q-factor. Thus we can conclude that far from bistability, the saturable absorber model used in the present calculations is equivalent to the non-resonant pump approach shortly introduced in section II.

- In the case of a short carrier lifetime: $T_{1} \ll \tau_{a 0}$, the carrier density can be adiabatically eliminated and the Q-factor enhancement only comes from the nonlinear behavior of the cavity. The $\mathrm{Q}$-factor reads

$$
Q=\frac{\omega_{0} \tau_{a 0}\left\{[\xi(x)+x]^{2}+x^{2}\right\}}{2(\eta-y)|\xi(x)|[\xi(x)+x]} .
$$


In the limit of $\eta \approx 1$ and $x \approx 1, Q$ can be approximated by

$$
Q=\frac{2 \omega_{0} \tau_{a 0}}{|1-x|}
$$

the Q-factor enhancement is only brought about by the bistable effect controlled by the pump beam. Comparing Eq. (40) and Eq. (43), we can evaluate the Q-factor enhancement coming from the carrier oscillations which is about $T_{1} /\left(4 \tau_{a 0}\right)$ for a given unsaturated absorption.

\section{Dispersive regime: $\alpha_{H} \gg 1$}

The cavity nonlinearity is thus mainly dispersive. In this case, we can consider that $x \ll 1$ and consequently $y \approx x$. We define $\Delta_{0}=\Delta \tau_{a 0}$ and $X=\alpha_{H} x$. These assumptions lead to the following expression for the reflection coefficient

$$
r(\delta)=\frac{Z(\delta)}{\vartheta(X)-X^{2}+j \delta T_{1} \vartheta(X)}
$$

where we have defined

$$
\begin{aligned}
\vartheta(X)= & \eta^{2}+\left(\Delta_{0}+2 X\right)^{2} \\
Z(\delta)= & \frac{2 \tau_{a 0}}{\tau_{e}}\left[\eta-\delta T_{1}\left(\Delta_{0}+X\right)+\right. \\
& \left.j\left(\Delta_{0}+2 X+\eta \delta T_{1}\right)\right] .
\end{aligned}
$$

Assuming $\delta T_{1} \ll 1$ and $\left|\vartheta(X)-X^{2}\right| \ll 1$, the reflection coefficient has a Lorentzian shape and the overall Q-factor is given by

$$
Q=\frac{\omega_{0} T_{1} \vartheta(X)}{2\left|\vartheta(X)-X^{2}\right|}
$$

The Q-factor enhancement mechanism is the same as in the absorptive case. As it has been underlined in the absorptive case, the Q-factor is proportional to the population lifetime $T_{1} . \vartheta(X)$ is strictly positive and thus never goes to zero. Nevertheless assuming $\Delta_{0}<-\sqrt{3} \eta$, which is the dispersive bistability condition for the pump [60], the equation $\vartheta(X)=X^{2}$ can have two solutions $X_{1}=\alpha_{H} x_{1}$ and $X_{2}=\alpha_{H} x_{2}$. When the normalized intracavity pump energy is close to one of these two values the Q-factor is strongly increased due to the nonlinear phase-shift and the differential amplification. This will be illustrated at section $\mathrm{V}$.

As a conclusion, this analytical study emphasizes the physical effects producing a strong Q-factor enhancement in a nonlinear microcavity. Let us emphasize that the cavity has a nonlinear behavior only for the pump beam which induces a strong dispersion on the probe beam via the saturation of the absorption and the population oscillations or the cross nonlinear index. The latter effect increases the dispersion at the probe wavelength thanks to the stiff phase shift undergone by the probe near the transition points of the bistable cavity transfer function.

\section{NUMERICAL RESULTS}

We note $Q_{\text {rad }}=\omega_{0} \tau_{\text {rad }} / 2$ and $Q_{e}=\omega_{0} \tau_{e} / 4$ respectively the radiative and external coupling Q-factors. Without absorption, for a linear microcavity, the maximal attainable $\mathrm{Q}$-factor is given by $1 /\left(Q_{\text {rad }}^{-1}+Q_{e}^{-1}\right)$. The associated normalized reflection is $\left(\frac{Q_{\text {rad }}}{Q_{\text {rad }}+Q_{e}}\right)^{2}$. All the following results have been obtained in a cavity with radiative losses such as $\tau_{\text {rad }}=\tau_{e}$.

\section{A. Absorptive nonlinearity}

In this section we consider a medium with a strong absorption and negligible nonlinear dispersive effects on the pump $\left(\alpha_{H}=0\right)$. In order to reach the pump induced bistability regime we assume that $\eta=1.05$. The aim of this study is to show that the induced dispersion can be used to increase the probe Q-factor. For this purpose we have considered a long carrier lifetime such as $T_{1}=$ $250 \tau_{a 0}$.

\section{Probe reflectivity spectrum}

Here, we focus on the case where a pump field is set at the cavity resonance $\left(\omega_{P}=\omega_{0}\right)$. Three reflection spectra are depicted Fig. 4.(a) for three different values of $x$. The dash curve is obtained for $x=0$, the resulting overall Q-factor is equal to $Q_{0}$ and the reflection under this condition is very low $\left(<10^{-3}\right)$ since $\tau_{a 0} \ll \tau_{e}$. The reflection spectrum displays a very narrow peak when $x$ increases $(x=0.1$ and $x=0.3$ ) mainly due to the fact that a strong dispersion is induced by the pump via the carrier oscillations. In the meantime, the absorption saturation increases the photon lifetime in the cavity $\tau$ and both effects sum up to improve the reflected power. These two effects were expected according to the basic model description at section II. The bistability does not play any role here since the chosen values of $x$ are sufficiently far from the turning points $\left(x_{1}\right.$ and $\left.x_{2}\right)$ as shown Fig. 4.(b). Next to the switching zone of the bistable power curve, the probe can be amplified. At Fig. 4.(c) the normalized intracaviy energy is set close to the first turning point $\left(x_{1}\right)$ in order to obtain $100 \%$ reflectivity. This spectrum is compared with the spectrum of an identical non absorbant cavity $(\alpha=0)$. Still slightly increasing the pump input power $(x=1.24)$ we do not only observe a strong Q-factor enhancement $\left(2.5 \times 10^{4} Q_{0}\right.$ instead of $20 Q_{0}$ in the best linear configuration obtained for $\alpha=0$ ) but also a selective amplification as depicted in Fig. 4.(d).

\section{Frequency pulling}

Frequency pulling, one of the main advantages of the configuration discussed in this paper, has not been tack- 

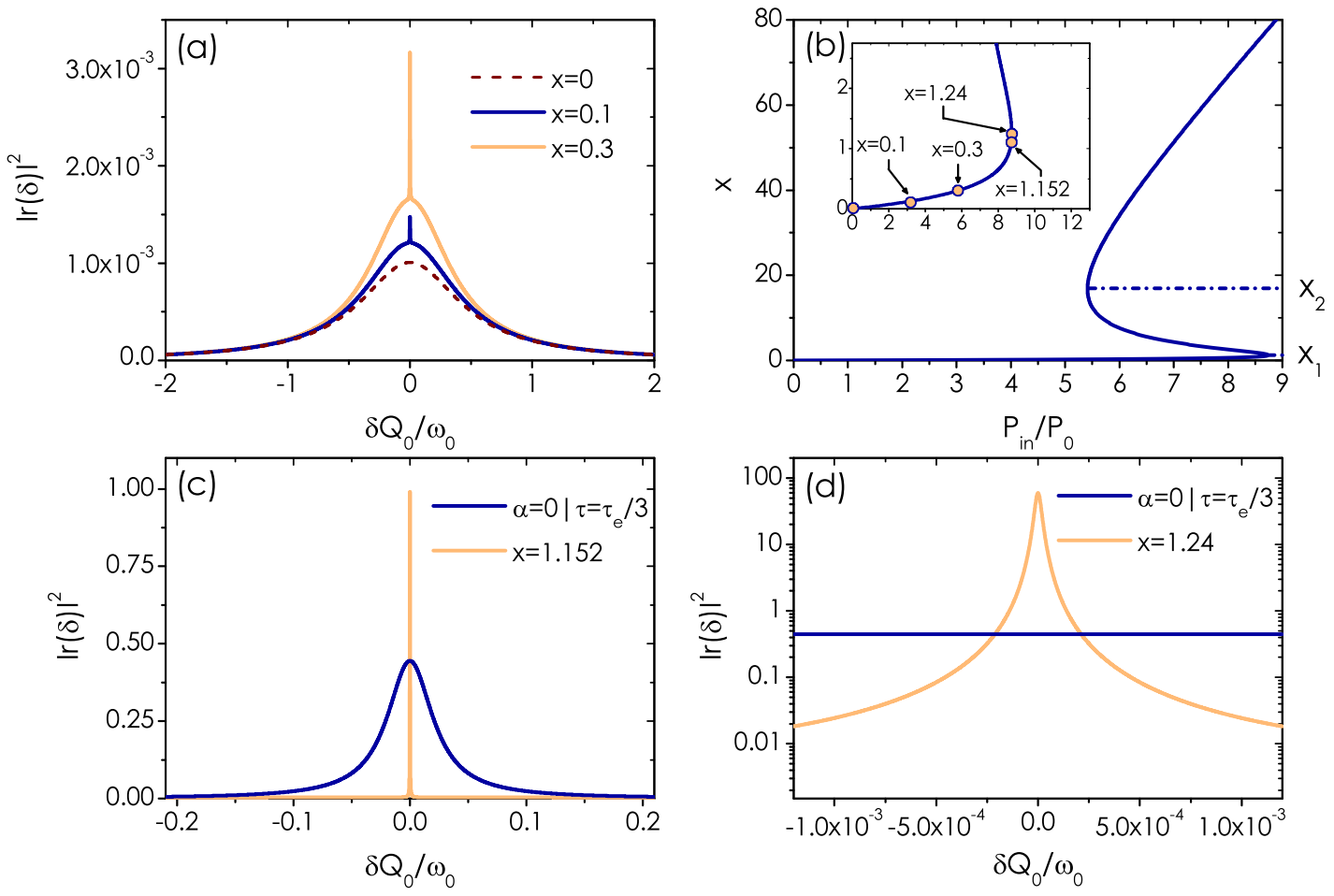

FIG. 4. (Color online) (a) Probe reflection spectra for three values of $x$ ( $x=0$ in dash line, $x=0.1$ in full dark line and $x=0.3$ in full light line) for a nonlinear cavity with $\eta=1.05, \alpha_{H}=0$ and $T_{1}=250 \tau_{a 0}$. (b) Intracavity normalized pump energy as a function of the input pump power. The inset is a zoom of the lowest part of the curve. (c) Probe reflection spectra, the value of $x=1.152$ has been set to obtain a 100\% reflection (light line). The case $\alpha=0$ (dark line) coresponds to an absorption-free linear cavity. (d) Example of a reflection spectrum with a high differential selective amplification obtained for $x=1.24$ (light line).

led yet. Figure 5 shows reflection spectra calculated for five decreasing values of the detuning $\Delta$ from $0.2 \omega_{0} / Q_{0}$ to $-0.2 \omega_{0} / Q_{0}$ where the intracavity energy is $x=1.24$. In this figure the arrow points out the cavity resonance. Whatever the value of the detuning may be, the probe resonance is pulled towards the pump frequency and its resonance is obtained at $\omega_{s} \approx \omega_{P}(\delta \approx 0)$. This effect has already been discussed for EIT [20]. Figure 5 also shows that the reflectivity decreases with a broader cavity/pump detuning. This can be compensated for by increasing the pump power. Figure 6 depicts the same conditions for a normalized intracavity energy and a pump detuning respectively set at: $x=1.42$ and $\Delta=-\omega_{0} /\left(15 Q_{0}\right)$. The reflectivity coefficient is about $100 \%$ and the resonance is at least two orders of magnitude narrower than the cavity/pump detuning.

\section{Role of population oscillations}

In the previous calculations we have only assumed a long carrier lifetime. In this paragraph we compare the reflectivity coefficients in the cases where $T_{1} \gg \tau_{a 0}$ and $T_{1} \ll \tau_{a 0}$. In the first case, the Q-factor enhancement is due to a combination of population oscillations and nonlinear effects whereas in the second case, the Q-factor enhancement is exclusively related to the pump bistability effects. At Fig. 7 we have plotted the Q-factor enhancement in comparison with the unsaturated cavity (Q-factor $Q_{0}$ ) both for $T_{1}=250 \tau_{a 0}$ and $T_{1} \ll \tau_{a 0}$. For $x \leq 0.2$ and $T_{1}=250 \tau_{a 0}$, the Q-factor increase is mainly due to population oscillations and for $x \geq 0.2$ the nonlinear effect induces an additional improvement. The straight horizontal dash line is the Q-factor of the cavity without absorption. For high values of $T_{1}$, the dispersion induced by the pump strongly rises the Q-factor in comparison with the short lifetime carrier approach. For example, in the case $T_{1}=250 \tau_{a 0}, Q=1 /\left(Q_{e}^{-1}+Q_{\text {rad }}^{-1}\right)$ for $P_{i n}=2.1 P_{0}(x=0.08)$ whereas in the limit of very 

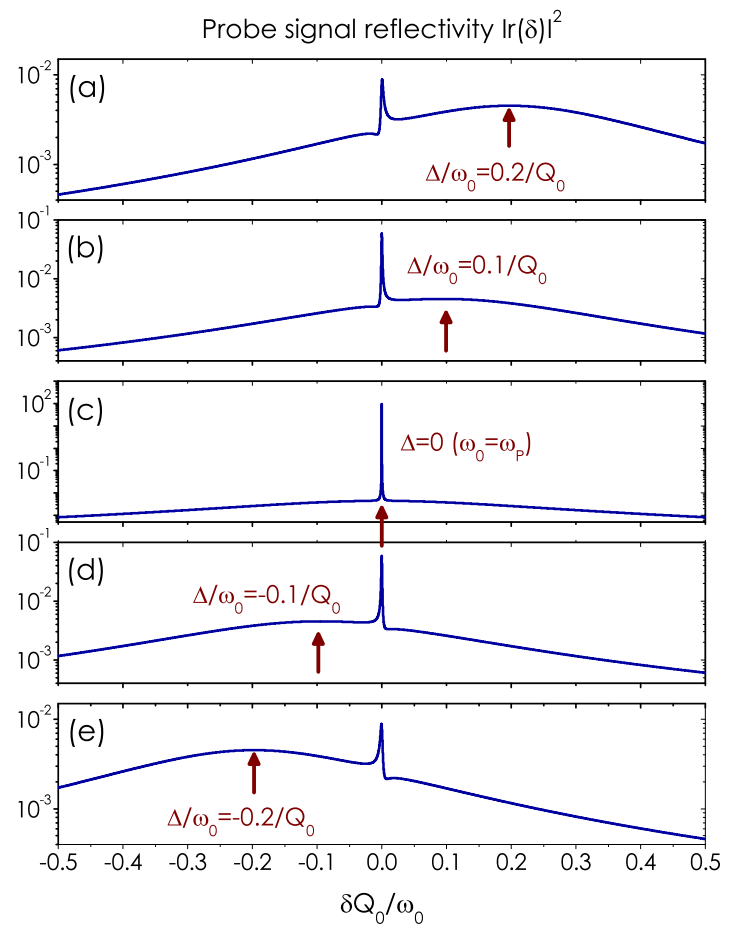

FIG. 5. (Color online) Probe reflection spectra for several values of cavity/pump detuning (a) $\Delta / \omega_{0}=0.2 / Q_{0}$ (b) $\Delta / \omega_{0}=0.1 / Q_{0}$ (c) $\Delta=0$ (d) $\Delta / \omega_{0}=-0.1 / Q_{0}$ e) $\Delta / \omega_{0}=-0.2 / Q_{0}$. In all the cases $x=1.24$ and the arrow points out the cavity resonances.

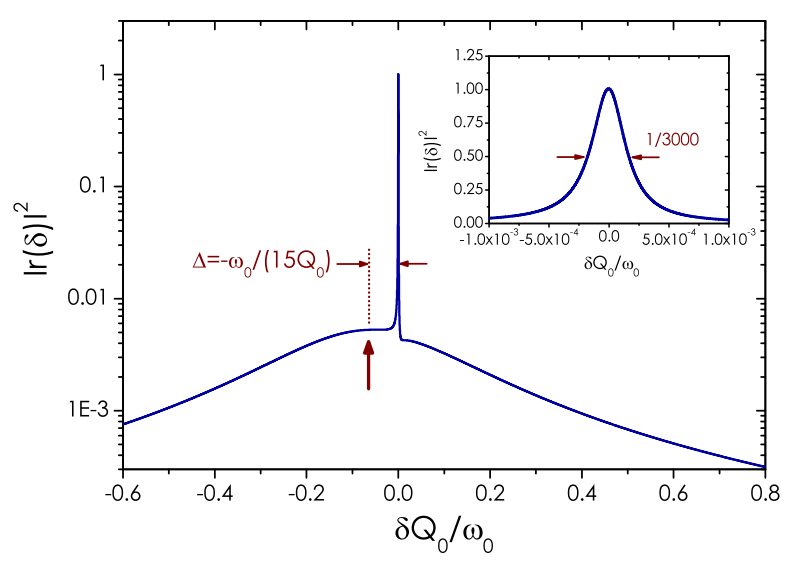

FIG. 6. (Color online) Probe reflection spectra for $\Delta=$ $-\omega_{0} /\left(15 Q_{0}\right)$ and $x=1.42$ in a logarithmic scale. The inset depicts the resonance with a linear scale. The resonance full width at half maximum is $\omega_{0} /\left(3000 Q_{0}\right)$.

short $T_{1}$, an input power $P_{i n}=8.6 P_{0}(x=1.02)$ is re-

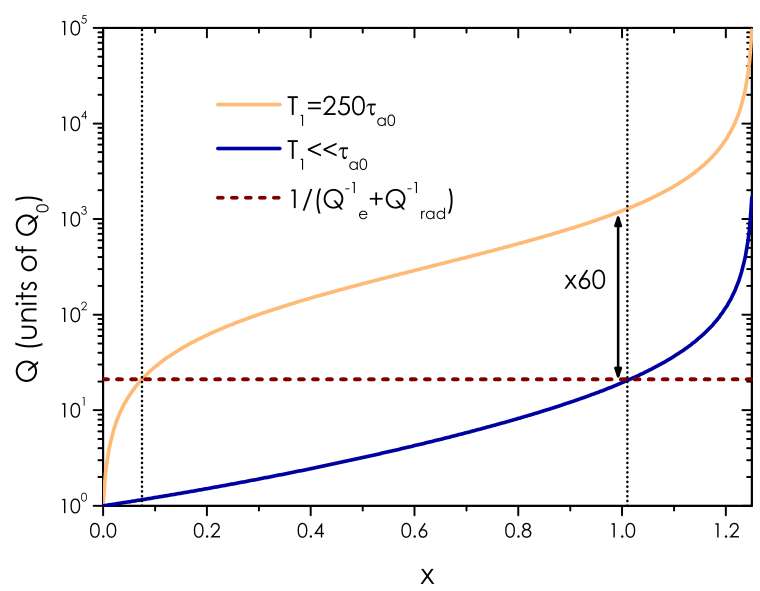

FIG. 7. (Color online) Quality factor for the probe signal as a function of the normalized pump intracavity energy $x$ for $T_{1}=250 \tau_{a 0}$ (light line) and $T_{1} \ll \tau_{a 0}$ (dark line). We have also plotted the value of the Q-factor of an equivalent linear cavity without absorption (dash line).

quired to obtain the same enhancement. At this power value the Q-factor is 60 times larger at $T_{1}=250 \tau_{a 0}$ than that at $T_{1} \ll \tau_{a 0}$. These results highlight the convenient effects of the population oscillations induced dispersion. Firstly it produces an increase factor around $T_{1} /\left(4 \tau_{a 0}\right)$ and secondly it reduces the required power for a given Q-factor enhancement. This enables us to use an input power much lower than the switching power. Note that near the switching point, the system is strongly sensitive to pump fluctuations as it is the case for delay lines based on critical slowing down $[61,62]$. As a consequence the system combining CPO and nonlinear effects is less sensitive to pump fluctuations and thus much more stable than purely nonlinear system where $T_{1} \ll \tau_{a 0}$.

\section{B. Dispersive nonlinearity}

We now consider a dispersive medium with $\alpha_{H}=25$. We have chosen $\eta=3$ to avoid absorptive bistability. The cavity/pump detuning is set to $\Delta_{0}=-3 \sqrt{3} \eta / 2$ in order to produce dispersive bistability. In most of the cases presented in this paragraph we have chosen $T_{1}=100 \tau_{a 0}$. Figure 8.(a) shows the reflectivity spectrum for $x=0.14$; we compare this spectrum with those without pumping $(x=0)$ or without absorption $(\alpha=0)$. As already extensively discussed in the absorption dominated case, the cavity resonance width is strongly reduced and next to the switching point as shown in Fig. 8.(b) the reflection signal can be increased $\left(|r(0)|^{2}=1\right.$ for $x=0.14)$ in comparison with the absorption-free cavity case $\left(|r(0)|^{2}=4 / 9\right)$. In Fig. 8.(c) the reflectivity spectra for $x=0.14$ and $x=0.156$ are displayed on a smaller 

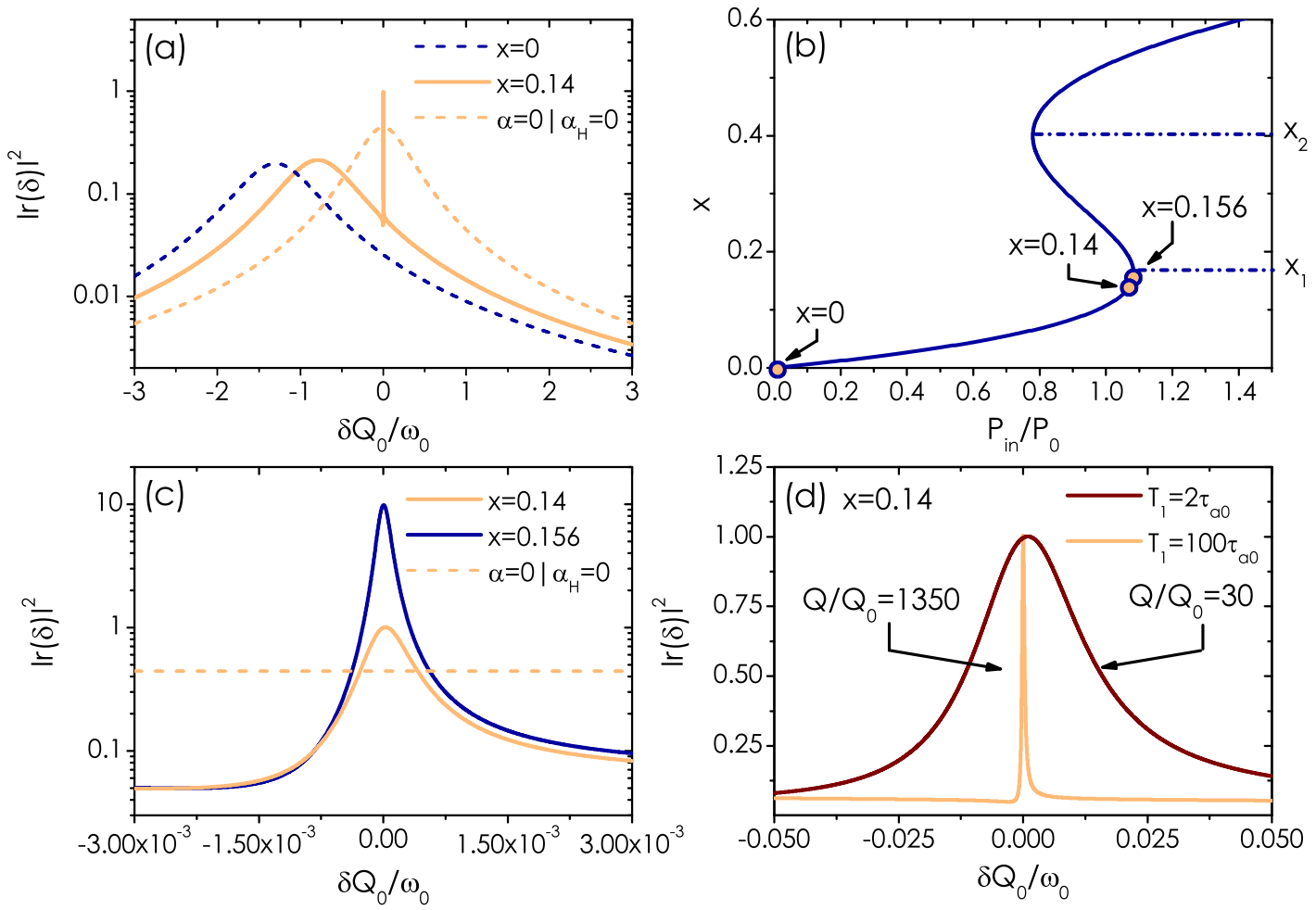

FIG. 8. (Color online) (a) Probe reflection spectra for $x=0$ (dash dark line) and $x=0.14$ (full line) in the case of a nonlinear cavity which physical characteristics are $\eta=3, \Delta_{0}=-3 \sqrt{3} \eta / 2, \alpha_{H}=25$ and $T_{1}=100 \tau_{a 0}$. For $x=0.14$, the cavity reflection is $100 \%$. For comparison, the bright dash line represents the spectrum of the same cavity in the linear and absorption-free case with $\alpha=0$ (dash light line). (b) Intracavity normalized pump energy as a function of the input pump power. (c) Probe reflection spectra for $x=1.4$ (light line) and 0.156 (full line). (d) Reflectivity spectrum calculated for $x=0.14$ assuming $T_{1}=100 \tau_{a 0}$ (light line) or $T_{1}=2 \tau_{a 0}$ (dark line).

frequency span. For $x=0.14$ the Q-factor enhancement is 1350. For $x=0.156$ the enhancement is around 5000 and the resonant reflectivity is close to 10. Finally in Fig. 8.(d) the reflectivity spectrum has been plotted again for $x=0.14$ and $T_{1}=100 \tau_{a 0}$ but also for $T_{1}=2 \tau_{a 0}$. We found a ratio of 45 between the two Q-factor enhancement values which is in good agreement with Eq. (46).

\section{CONCLUSION}

We have proposed a simple approach, which can be analytically solved, to describe population oscillations in a nonlinear microcavity. We consider a generic TLS (semiconductor, atomic medium, ...) driven by a powerful pump field and probed by a weak signal. The analytical model enables us to discuss the physical processes which lead both to a strong enhancement of the Q-factor and to a control of the transmission at the probe frequency. The simultaneous action of population oscilla- tions and the nonlinear response of the cavity induces a strong intracavity dispersion and a differential gain. This technique could be used to stabilize a microcavity using the frequency pulling effect for example. Finally, it is also possible to take advantage of the active control of the Q-factor via the pump beam in optical pulse buffering applications [7]. Note that most of the parameters used for the numerical simulations can be reached for usual semiconductor nanocavities. We have recently demonstrated both Q-factor enhancement and frequency pulling at $1.55 \mu \mathrm{m}$ in a single mode L3 photonic crystal semiconductor nanocavity containing four quantum wells as driven TLS.

\section{ACKNOWLEDGMENTS}

We acknowledge support by the French Agence Nationale de la Recherche through project CALIN (ANR 2010 BLAN-1002) and project ORA (ANR 2010 BLAN- 
0312). These results are within the scope of C'nano IdF and RTRA Triangle de la Physique; C'nano IdF is a CNRS, CEA, MESR and Région Ile-de-France Nanosciences Competence Center.
[1] K. J. Vahala, Nature (London), 424, 839 (2003).

[2] A. Matsko and V. Ilchenko, IEEE J. Sel. Top. Quantum Electron., 12, 3 (2006).

[3] V. Ilchenko and A. Matsko, IEEE J. Sel. Top. Quantum Electron., 12, 15 (2006).

[4] D. Strekalov, D. Aveline, N. Yu, R. Thompson, A. Matsko, and L. Maleki, IEEE J. Lightwave Technol., 21, 3052 (2003).

[5] A. N. Oraevsky, A. V. Yarovitsky, and V. L. Velichansky, Quantum Electron., 31, 897 (2001).

[6] T. Tanabe, M. Notomi, E. Kuramochi, A. Shinya, and H. Taniyama, Nature Photon., 1, 49 (2007).

[7] M. F. Yanik and S. Fan, Phys. Rev. Lett., 92, 083901 (2004).

[8] Q. Xu, P. Dong, and M. Lipson, Nature Phys., 3, 406 (2007).

[9] Y. Dumeige, Europhys. Lett., 86, 14003 (2009).

[10] A. A. Savchenkov, V. S. Ilchenko, A. B. Matsko, and L. Maleki, Phys. Rev. A, 70, 051804 (2004).

[11] Y. Takahashi, H. Hagino, Y. Tanaka, B.-S. Song, T. Asano, and S. Noda, Opt. Express, 15, 17206 (2007).

[12] Y. Akahane, T. Asano, B. Song, and S. Noda, Nature (London), 425, 844 (2003).

[13] J. Lu and J. Vuckovic, Opt. Express, 18, 3793 (2010).

[14] S. Minin, M. R. Fisher, and S. L. Chuang, Appl. Phys. Lett., 84, 3238 (2004).

[15] P. Peng, C. Lin, H. Kuo, G. Lin, W. Tsai, H. Yang, K. Lin, J. Chi, S. Chi, and S. Wang, Electron. Lett., 42, 1036 (2006).

[16] Y. Dumeige, S. Trebaol, L. Ghişa, T. K. N. Nguyên, H. Tavernier, and P. Féron, J. Opt. Soc. Am. B, 25, 2073 (2008).

[17] M. Soljačić, E. Lidorikis, L. V. Hau, and J. D. Joannopoulos, Phys. Rev. E, 71, 026602 (2005).

[18] G. Müller, M. Müller, A. Wicht, R.-H. Rinkleff, and K. Danzmann, Phys. Rev. A, 56, 2385 (1997).

[19] T. Lauprêtre, C. Proux, R. Ghosh, S. Schwartz, F. Goldfarb, and F. Bretenaker, Opt. Lett., 36, 1551 (2011).

[20] M. D. Lukin, M. Fleischhauer, M. O. Scully, and V. L. Velichansky, Opt. Lett., 23, 295 (1998).

[21] H. Wang, D. J. Goorskey, W. H. Burkett, and M. Xiao, Opt. Lett., 25, 1732 (2000).

[22] D. J. Goorskey, H. Wang, W. H. Burkett, and M. Xiao, J. Mod. Opt., 49, 305 (2002).

[23] W. Yang, A. Joshi, and M. Xiao, Opt. Lett., 29, 2133 (2004).

[24] H. Wu and M. Xiao, Opt. Lett., 32, 3122 (2007).

[25] S. Weis, R. Rivière, S. Deléglise, E. Gavartin, O. Arcizet, A. Schliesser, and T. J. Kippenberg, Science, 330, 1520 (2010).

[26] M. S. Bigelow, N. N. Lepeshkin, and R. W. Boyd, Phys. Rev. Lett., 90, 113903 (2003).

[27] M. S. Bigelow, N. N. Lepeshkin, and R. W. Boyd, Science, 301, 200 (2003).

[28] E. Baldit, K. Bencheikh, P. Monnier, J. A. Levenson, and V. Rouget, Phys. Rev. Lett., 95, 143601 (2005).
[29] P. C. Ku, C. J. Chang-Hasnain, and S. L. Chuang, J. Phys. D: Appl. Phys., 40, R93 (2007).

[30] X. Zhao, P. Palinginis, B. Pesala, C. Chang-Hasnain, and P. Hemmer, Opt. Express, 13, 7899 (2005).

[31] N. Laurand, S. Calvez, M. D. Dawson, and A. E. Kelly, Opt. Express, 14, 6858 (2006).

[32] V. S. C. Manga Rao and S. Hughes, Opt. Lett., 32, 304 (2007).

[33] C.-S. Chou, R.-K. Lee, P.-C. Peng, H.-C. Kuo, G. Lin, H.-P. Yang, and J. Y. Chi, J. Opt. A: Pure Appl. Opt., 10, 044016 (2008).

[34] T. R. Nielsen, A. Lavrinenko, and J. Mork, Appl. Phys. Lett., 94, 113111 (2009).

[35] M. R. Singh, Opt. Lett., 34, 2909 (2009).

[36] A. El Amili, B.-X. Miranda, F. Goldfarb, G. Baili, G. Beaudoin, I. Sagnes, F. Bretenaker, and M. Alouini, Phys. Rev. Lett., 105, 223902 (2010).

[37] H. M. Gibbs, S. L. McCall, and T. N. C. Venkatesan, Phys. Rev. Lett., 36, 1135 (1976).

[38] H. A. Haus, Waves and Fields in Optoelectronics (Prentice-Hall, 1984).

[39] Q. Li, T. Wang, Y. Su, M. Yan, and M. Qiu, Opt. Express, 18, 8367 (2010).

[40] R. W. Boyd, M. G. Raymer, P. Narum, and D. J. Harter, Phys. Rev. A, 24, 411 (1981).

[41] C. Sauvan, P. Lalanne, and J. P. Hugonin, Phys. Rev. B, 71, 165118 (2005).

[42] Y. Dumeige, S. Trebaol, and P. Féron, Phys. Rev. A, 79, 013832 (2009)

[43] V. Zapasskii and G. Kozlov, Opt. Spectrosc., 100, 419 (2006).

[44] G. Piredda and R. W. Boyd, J. Europ. Opt. Soc. Rap. Public., 2, 07004 (2007).

[45] B. Macke and B. Ségard, Phys. Rev. A, 78, 013817 (2008).

[46] A. Selden, Opt. Spectrosc., 106, 881 (2009).

[47] K. Bencheikh, E. Baldit, S. Briaudeau, P. Monnier, J. A. Levenson, and G. Mélin, Opt. Express, 18, 25642 (2010).

[48] M. F. Yanik, S. Fan, and M. Soljačić, Appl. Phys. Lett., 83, 2739 (2003).

[49] Y. Dumeige, C. Arnaud, and P. Féron, Opt. Commun., 250, 376 (2005).

[50] A. De Rossi, M. Lauritano, S. Combrié, Q. V. Tran, and C. Husko, Phys. Rev. A, 79, 043818 (2009).

[51] S. Balle, Opt. Commun., 119, 227 (1995).

[52] C. Henry, IEEE J. Quantum Electron., 18, 259 (1982).

[53] L. Spinelli, G. Tissoni, M. Brambilla, F. Prati, and L. A. Lugiato, Phys. Rev. A, 58, 2542 (1998).

[54] A. M. Yacomotti, F. Raineri, C. Cojocaru, P. Monnier, J. A. Levenson, and R. Raj, Phys. Rev. Lett., 96, 093901 (2006).

[55] A. M. Yacomotti, P. Monnier, F. Raineri, B. B. Bakir, C. Seassal, R. Raj, and J. A. Levenson, Phys. Rev. Lett., 97, 143904 (2006).

[56] M. Brunstein, Ph.D. thesis, Université Paris-Sud XI, France (2011). 
[57] E. by Eli Kapon, Semiconductor laser I: Fundamentals, edited by A. Press (1999).

[58] F. Raineri, A. M. Yacomotti, T. J. Karle, R. Hostein, R. Braive, A. Beveratos, I. Sagnes, and R. Raj, Opt. Express, 17, 3165 (2009).

[59] R. W. Boyd, Nonlinear Optics (Academic Press, 2008).
[60] H. M. Gibbs, Optical Bistability: Controlling Light With Light (Academic Press, 1985).

[61] R. Bonifacio and P. Meystre, Opt. Commun., 29, 131 (1979).

[62] A. Daunois and J. Y. Bigot, Appl. Phys. B, 45, 157 (1988). 